\title{
Mechanical Modulation of the Solid-State Luminescence of Tricarbonyl Rhenium(I) Complexes Through the Interplay Between Two Triplet Excited States
}

\author{
Jan Patrick Calupitan, ${ }^{[a]}$ Alexandre Poirot ${ }_{,}^{[b]}$ Jinhui Wang $_{,}{ }^{[b, c, d]}$ Béatrice Delavaux-Nicot, ${ }^{[e]}$ \\ Mariusz Wolff, ${ }^{[f]}$ Maria Jaworska, ${ }^{[g]}$ Rémi Métivier, ${ }^{[a]}$ Eric Benoist, ${ }^{[b]}$ Clémence Allain, ${ }^{*[a]}$ and \\ Suzanne Fery-Forgues ${ }^{*[b]}$
}

Abstract: Mechanoresponsive luminescence (MRL) materials promise smart devices for sensing, optoelectronics and security. We present here the first report on the MRL activity of two Re' complexes, opening up new opportunities for applications in these fields. Both complexes exhibit marked solidstate luminescence enhancement (SLE). Furthermore, the pristine microcrystalline powders emit in the yellow-green region, and grinding led to an amorphous phase with concomitant emission redshift and shrinking of the photoluminescence $(\mathrm{PL})$ quantum yields and lifetimes. Quantum chemical calculations revealed the existence of two low-lying triplet excited states with very similar energy levels, that is, ${ }^{3} \mathrm{IL}$ and ${ }^{3} \mathrm{MLCT}$, having, respectively, almost pure intraligand (IL) and metal-to-ligand charge-transfer (MLCT) character. Transi- tion between these states could be promoted by rotation around the pyridyltriazole-phenylbenzoxazole bond. In the microcrystals, in which rotations are hindered, the ${ }^{3} \mathrm{IL}$ state induces the prominent PL emission at short wavelengths. Upon grinding, rotation is facilitated and the transition to the ${ }^{3} \mathrm{MLCT}$ state results in a larger proportion of long-wavelength PL. FTIR and variable-temperature PL spectroscopy showed that the opening of the vibrational modes favours non-radiative deactivation of the triplet states in the amorphous phase. In solution, PL only arises from the ${ }^{3} \mathrm{MLCT}$ state. The same mechanism accounts for the spectroscopic differences observed when passing from crystals to amorphous powders, and then to solutions, thereby clarifying the link between SLE and MRL for these complexes. [a] Dr. J.P. Calupitan, Dr. R. Métivier, Dr. C. Allain Laboratoire PPSM, CNRS UMR 8531, ENS Paris-Saclay Université Paris-Saclay, 91190 Gif-sur-Yvette (France) E-mail:callain@ens-paris-saclay.fr

[b] A. Poirot, Dr. J. Wang, Prof. E. Benoist, Dr. S. Fery-Forgues SPCMIB, CNRS UMR5068

Université Toulouse III-Paul Sabatier

118 route de Narbonne, 31062 Toulouse Cedex 9 (France)

E-mail: sff@chimie.ups-t/se.fr

[c] Dr. J. Wang

Institute of Drug Discovery Technology Ningbo University, $\mathbf{\square}$, Ningbo 315211 (P.R. China) Please give street name and number for affiliations $c$ and $d . \mathbf{\square}$

[d] Dr. J. Wang

State Key Laboratory of Chemical Oncogenomics

Key Laboratory of Chemical Biology, The Graduate School at Shenzhen Tsinghua University

घE, Shenzhen, Guangdong 518055 (P.R. China)

[e] Dr. B. Delavaux-Nicot

Laboratoire de Chimie de Coordination, CNRS (UPR 8241)

Université de Toulouse (UPS, INPT), 205 route de Narbonne, 31077 Toulouse Cedex 4 (France)

[f] Dr. M. Wolff

Institut für Chemische Katalyse

Universität Wien, Währinger Strasse 38, 1090 Wien (Austria)

[g] Dr. M. Jaworska

Institute of Chemistry, University of Silesia 9th Szkolna St., 40-006 Katowice (Poland)

Supporting information and the ORCID identification number(s) for the au-

(iD thor(s) of this article can be found under: https://doi.org/10.1002/chem.202005245.

\section{Introduction}

Mechanoresponsive luminescence (MRL), the change in emission properties upon application of an external mechanical stimulus, has attracted considerable attention in recent years for smart material applications. ${ }^{[1-12]}$ MRL is a supramolecular phenomenon, as it coincides with transitions between two crystalline polymorphs or between crystalline and amorphous states: emission changes are generally a result of the modification of the molecular environment during these transitions. ${ }^{[1,2]}$ In recent years, MRL-active organic luminogens have been widely reported ${ }^{[3-6]}$ in the context of aggregation-induced emission (AIE), ${ }^{[3,4]}$ that is, for molecules that are weakly or nonemissive in dilute solutions, but strongly luminescent upon aggregate formation. The rationale is that AIE-active molecules, which are generally non-planar, tend to pack loosely, and so crystals are readily destroyed by weak mechanical stress.

Meanwhile, MRL-active transition-metal complexes, ${ }^{[7]}$ which present alternative emission pathways, have been relatively under-researched, even though they promise applications in security, ${ }^{[7-9]}$ data storage ${ }^{[7,9]}$ and pressure/force sensing. ${ }^{[10]}$ Recently, we introduced new tricarbonyl rhenium(I) complexes $^{[13]}$ that show rare $\mathrm{AIE}$, and more widely speaking, solid-state luminescence enhancement (SLE). ${ }^{[14]}$ Drawing lessons and parallels from organic AIE- and MRL-active materials, we sought to explore the MRL response of our $\operatorname{Re}^{\prime}$ complexes. MRL has been 
reported for a variety of transition-metal complexes, including

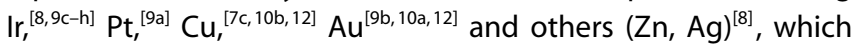
are frequently also AIE-active. However, to the best of our knowledge, this is the first observation of MRL in $\mathrm{Re}^{\prime}$ complexes. Furthermore, the mechanism proposed for their activity sheds new light on the SLE process.

\section{Results and Discussion}

The rhenium complexes 1 and 2 (Figure 1) contain ligands consisting of a 3-(2-pyridyl)-1,2,4-triazole (pyta) fragment appended to a 2-phenylbenzoxazole (PBO) moiety. They differ in the connection between the $\mathrm{PBO}$ and pyta fragments, and by the presence of a bulky tert-butyl group in complex 1 . Both were selected for study as a result of their excellent emission properties in the solid state. ${ }^{[13 b]}$ Upon irradiation with near-UV light or low-wavelength visible light, pristine powders of $\mathbf{1}$ and $\mathbf{2}$, obtained by crystallisation in methanol, showed emission in the yellow and green regions, respectively, with high photoluminescence quantum yields (Table 1). It can be recalled that in dichloromethane, both compounds emitted weak red light. ${ }^{[13 b]}$ The SLE effect is conventionally attributed to the restriction of molecular motion upon aggregation.

Mechanical grinding of the rhenium complexes caused a redshift of emission by $30 \mathrm{~nm}\left(892 \mathrm{~cm}^{-1}\right)$ for 1 and $58 \mathrm{~nm}$ $\left(1783 \mathrm{~cm}^{-1}\right.$ ) for 2 (Figure 1 and Table 1) and the quantum yields collapsed. Upon exposure of the ground powders to solvent fumes (chloroform, dichloromethane or THF), the emission associated with the corresponding pristine powders was fully recovered. The process was reversible several times without any noticeable changes. The excitation spectra also showed variation in the intensity of the metal-to-ligand charge transfer (MLCT) band (447 nm for 1, $437 \mathrm{~nm}$ for 2) for the pristine and ground forms. According to the 3D luminescence spectra, the emission spectral shape is independent of $\lambda_{\text {ex }}$ (see Figures S1 and S2 in the Supporting Information), which indi-
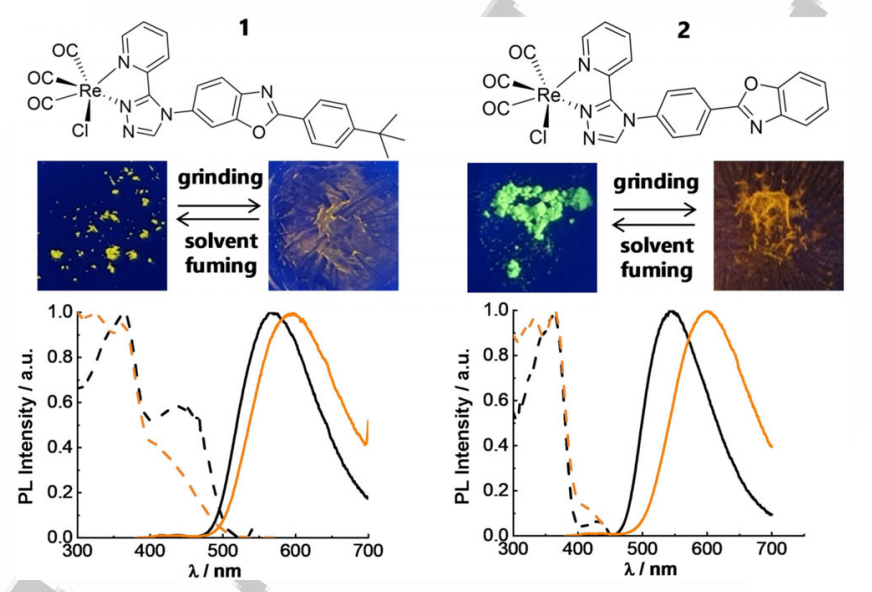

Figure 1. From top to bottom, for complexes 1 (left) and 2(right): the respective chemical structures, photographs of pristine and ground powders under UV light ( $365 \mathrm{~nm}$ ), and normalised excitation (dotted) and emission (solid) spectra of pristine (black) and ground forms (orange). $\lambda_{\mathrm{ex}}=365 \mathrm{~nm}$ for the emission spectra; the excitation spectra were recorded at the emission maxima.
Table 1. Phosphorescence properties of complexes $\mathbf{1}$ and $\mathbf{2}$ in the solid state (pristine and ground powders) and in dichloromethane solution. ${ }^{[a]}$

\begin{tabular}{|c|c|c|c|c|c|c|}
\hline & $\begin{array}{l}\lambda_{\max }^{\mathrm{em}} \\
{[\mathrm{nm}]^{[\mathrm{b}]}}\end{array}$ & $\Phi_{\mathrm{PL}}^{[c]}$ & $\begin{array}{l}\tau \\
{[\mathrm{ns}]^{[\mathrm{d}]}}\end{array}$ & $\begin{array}{c}\langle\tau> \\
{[\mathrm{ns}]^{[\mathrm{e}]}}\end{array}$ & $\begin{array}{l}k_{\mathrm{r}} \\
{\left[\mathrm{ns}^{-1}\right]^{[\mathrm{f}]}}\end{array}$ & $\begin{array}{l}k_{\mathrm{nr}} \\
{\left[\mathrm{ns}^{-1}\right]^{[\mathrm{g}]}}\end{array}$ \\
\hline \multicolumn{7}{|c|}{ Complex 1} \\
\hline pristine & 565 & 0.21 & $\begin{array}{c}890(0.76), 185 \\
(0.19)\end{array}$ & 710 & $3.0 \times 10^{5}$ & $1.1 \times 10^{6}$ \\
\hline ground & 595 & 0.05 & $214(0.71), 47(0.22)$ & 160 & $3.1 \times 10^{5}$ & $5.9 \times 10^{6}$ \\
\hline solution $^{[a]}$ & 627 & 0.017 & $\begin{array}{c}100 \\
\text { Complex } 2\end{array}$ & 100 & $1.7 \times 10^{5}$ & $9.8 \times 10^{6}$ \\
\hline pristine & 542 & 0.55 & $563(0.85), 93(0.12)$ & 490 & $1.1 \times 10^{6}$ & $9.2 \times 10^{5}$ \\
\hline ground & 600 & 0.25 & $260(0.82), 60(0.14)$ & 220 & $1.1 \times 10^{6}$ & $3.4 \times 10^{6}$ \\
\hline solution $^{[\mathrm{a}]}$ & 632 & 0.012 & 80 & 79 & $1.5 \times 10^{5}$ & $1.3 \times 10^{7}$ \\
\hline
\end{tabular}

[a] Concentration $\approx 3.5 \times 10^{-5} \mathrm{M}$, bubbled with $\mathrm{Ar}(5 \mathrm{~min}$ ) before measurement. From ref. [13b]. [b] Maximum emission wavelength. $\lambda_{\mathrm{ex}}=380 \mathrm{~nm}$. [c] Phosphorescence quantum yield. [d] Main lifetimes with fractions of intensity given in parentheses. [e] Weighted average lifetimes. [f] Radiative rate constants, $k_{\mathrm{r}}=\Phi_{\mathrm{PL}} /\langle\tau\rangle$. [g] Non-radiative rate constant, $k_{\mathrm{nr}}=$ $\left(1-\Phi_{\mathrm{PL}}\right) /<\tau>$.

cates the presence of a single emitting species for the pristine and ground forms of both compounds. Remarkably, each of the pristine powders displayed two main distinct luminescence lifetimes (890 and 185 ns for 1, 563 and 93 ns for 2), which were drastically shortened upon grinding (see Table S1, Figures S3 and S4 and further comments on lifetime measurements in the Supporting Information). Such values comprising several tens and several hundred nanoseconds confirm the phosphorescent nature of the emission.

Crystallographic analyses of the two compounds have been reported previously. ${ }^{[13 b]}$ Besides the expected octahedral geometry of the coordination sphere, other characteristic features include the planarity of the PBO moiety and its pronounced twist with respect to the 1,2,4-triazole moiety due to the repulsion of the hydrogen atoms in the adjacent rings. They exhibit dihedral angles of 72.5 and $62.8^{\circ}$ for the two molecules that constitute the unit cell of 1 , and $83.3^{\circ}$ for 2 (Figure 2). Furthermore, the aromatic systems of neighbouring molecules of the two complexes do not overlap in the respective crystal packing modes. The crystals, structured by various weak interactions, are therefore prone to fracture by external stimuli.

Powder X-ray diffraction (PXRD) analysis revealed the crystalline nature of the pristine powders, showing the same peaks as those simulated on the basis of the single-crystal X-ray diffraction data. By contrast, the ground samples generated
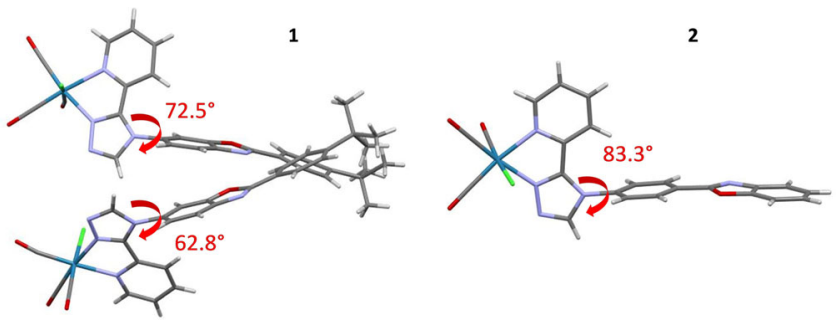

Figure 2. Conformations of complexes 1 (with two molecules in the asymmetric unit) and 2. The pyta-benzoxazole dihedral angles are shown. 
a
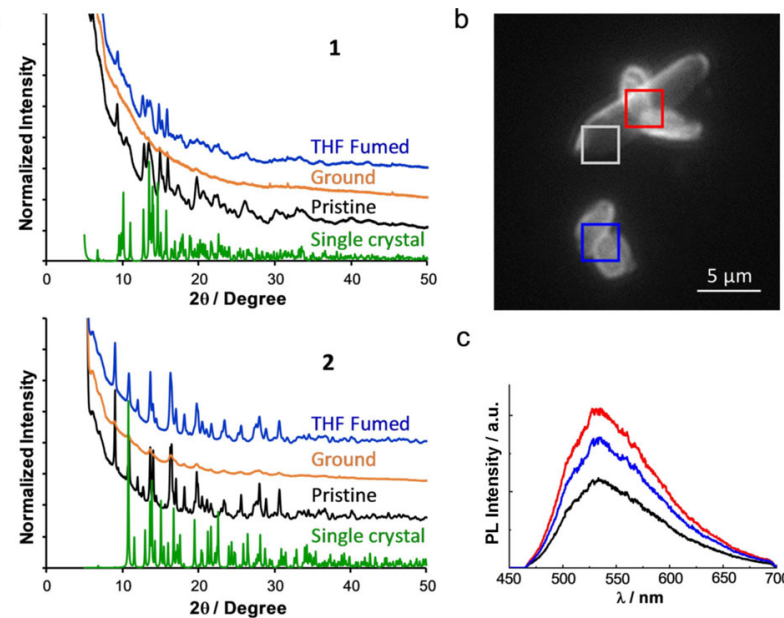

C

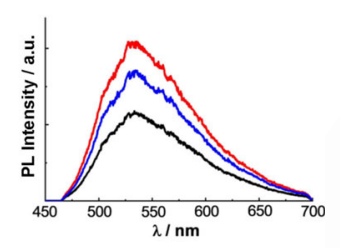

Figure 3. a) PXRD patterns of the pristine, ground and THF-fumed powders, and comparison with the data derived from $\mathrm{X}$-ray diffraction analysis of a single crystal of 1 (top) and $\mathbf{2}$ (bottom). b) Fluorescence microscopy image of microcrystals of 2 . $\lambda_{\mathrm{ex}}=400 \mathrm{~nm}$. c) The corresponding emission spectra. The colours of the spectra refer to the zone where the emission signal was taken, except for the white zone, which gives the black spectrum.

almost flat patterns. The samples exposed to solvent fumes exhibited the same peaks as the pristine powders (Figure $3 a$ ). This confirms the transition from crystalline pristine powders to an amorphous state upon grinding, and the recovery of the initial crystalline structure upon solvent fuming, which favours the mobility of molecules.

Coupled optical atomic force microscopy ${ }^{[16]} \square$ Ref. 15 does not appear to have been cited in the text. Please check. (AFM) imaging was performed to ascertain the origin of emission in the crystalline samples. After drop-deposition and drying of a dichloromethane solution of 2, AFM showed flat objects, typical of uniformly ordered crystalline structures (see Figure S5 in the Supporting Information). Collecting the light emitted by single crystals of $\mathbf{2}$ allowed us to record the corresponding spectra, which were in complete agreement with that of the pristine powder (Figure $3 b, c$ ).

The differences in the spectroscopic properties are therefore associated with phase changes from the crystalline to amorphous states. The lack of strong face-to-face $\pi-\pi$-stacking interactions in the crystals of $\mathbf{1}$ and $\mathbf{2}$ suggests that excimer formation or exciton coupling, widely evoked in the literature to explain MRL in AIE luminogens, ${ }^{[1,4,5,6 a, 7]}$ plays a minor role in our complexes. The rationale for the observed MRL effect must rather be researched on the molecular scale. It is commonly agreed that for typical propeller-shaped AIE-active compounds, which are often stuck in a twisted conformation in the crystalline state,${ }^{[1-7]}$ grinding releases the twist stress and allows molecules to adopt more planar conformations. These spectacular conformational changes usually $\square$ 'usually' inserted here to provide a context for the following sentence. Ok? $\square$ account for the large differences in spectroscopic properties. This is not the case here: the overall geometries of the molecules in the crystalline state, provided by XRD analyses, are similar to those of the molecules surrounded by a solvent continuum, optimised by density functional theory (DFT) calculations. ${ }^{[13 b]}$ The only significant difference for 1 is the value of the dihedral angle between the pyta and PBO moieties (calcd $89.4^{\circ}$ vs. exptl $72.5 / 62.8^{\circ}$, see Table S2 in the Supporting Information). Furthermore, for both compounds, it is notable that the dihedral angle decreases when passing from the ground state to the first excited state. For instance, for complex 2, the calculated dihedral angle for the ${ }^{3} \mathrm{MLCT}$ state is $55.5^{\circ}$, whereas the experimental angle is $83.3^{\circ}$ in the crystal. No clear trend could be observed for the two compounds, but our attention was attracted by the pyta-PBO dihedral angle. Furthermore, free rotation around the bond linking pyta and PBO is possible in solution.

Consequently, it seemed instructive to investigate the influence of molecular motion upon the energy of the first triplet state, which is thought to be responsible for emission. DFT calculations were performed. The effect of rotation around the pyta-PBO bond on the PL emission wavelength was calculated from the Boltzmann distribution average versus rotation angle at a temperature of $300 \mathrm{~K}$. The curves for potential energy plotted against pyta-PBO dihedral angle have almost identical shapes for compounds 1 (Figure S6 d in the Supporting Information) and 2 (Figure $4 \mathrm{a}$ ), with a large area of very low
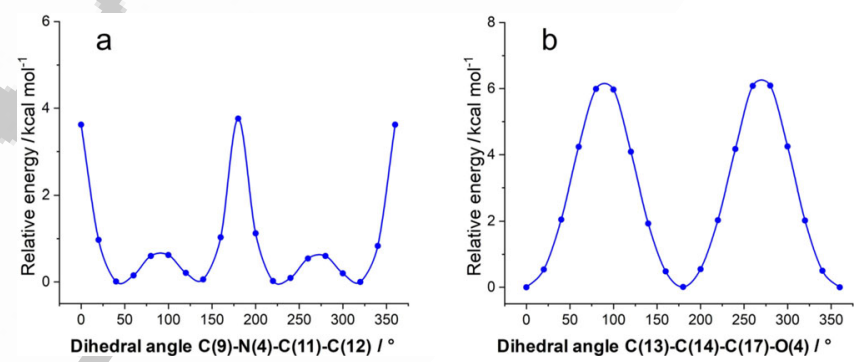
Dihedral angle $C(13)-C(14)-C(17)-O(4) l^{\circ}$

Figure 4. Potential energy curves for the lowest-energy triplet excited state of $\mathbf{2}$ versus the dihedral angle of a) the pyta-PBO bond and $b$ ) the internal $\mathrm{PBO}$ bond, calculated at the PBE1PBE/6-31+G** level of theory.

energy. Thus, rotation is thermally easy, with rotational barriers of 4.64 and $3.76 \mathrm{kcalmol}^{-1}$ for 1 and 2, respectively, and has only a small impact on the PL emission wavelength (see Tables S3, S5 and S9). The effect of internal rotation within the PBO moiety was also investigated, because no repulsion impedes the rotation of the phenyl group with respect to the benzoxazole group. In this case, the potential energy sharply increases when the dihedral angle deviates from 0 or $180^{\circ}$ (Figure $\mathrm{S} 6 \mathrm{f}$ and Figure $4 \mathrm{~b}$ ). The rotational barrier is somewhat higher than in the former case $\left(6.09\right.$ and $6.19 \mathrm{kcal} \mathrm{mol}^{-1}$ for 1 and 2, respectively, see Table 54 ), which suggests that this moiety is more likely to stay planar at room temperature. Most importantly, the effect of torsion on the PL emission is almost insignificant (see Tables S3, S7 and S10).

Further extensive DFT calculations were performed to gain an understanding of the MRL effect. In our previous work, PL emission was shown to arise from the first triplet state, which has strong MLCT character, as a result of the transfer of one electron from the $d(R e)$ orbital to the $\pi^{*}$ orbital of the organic ligand. This work allowed us to identify a second triplet state 
with intraligand (IL) character, namely ${ }^{3} \mathrm{IL}$, because the promotion of one electron occurs from the $\pi$ orbital to $a \pi^{*}$ orbital of the organic ligand (see Figures $S 6 b, c$ and $S 7 b, c$ in the Supporting Information). Indeed, the existence of two long-lived equilibrated triplet states has been reported previously by Vlček and Zàliš and co-workers for $\left[\operatorname{Re}(\mathrm{Cl})(\mathrm{CO})_{3}(\mathrm{~N}, \mathrm{~N})\right]$ complexes $(\mathrm{N}, \mathrm{N}=$ bipyridine, phenanthroline or 4,7-dimethylphenanthroline). Ultrafast spectroscopic measurements showed that excitation at $400 \mathrm{~nm}$ produces a ${ }^{1} \mathrm{CT}$ state, which decays on a femtosecond timescale and populates simultaneously the lowestlying phosphorescent triplet state with strong charge-transfer (CT) character and an intermediate triplet state with substantial IL character. The ${ }^{3} \mathrm{IL}-$ to- $^{3} \mathrm{CT}$ conversion occurs on a picosecond timescale, depending on the solvent and ligands. ${ }^{[17]}$ In this case, the calculated energy of the ${ }^{3} \mathrm{IL}$ state of 1 is higher than that of ${ }^{3} \mathrm{MLCT}$ by only about $5 \mathrm{kcal} \mathrm{mol}^{-1}(-1708.45268 \mathrm{a} . \mathrm{u}$. vs. -1708.46086 a.u.). For 2 , the energies of the two triplet states are very similar $(-1551.38412$ and -1551.38736 a.u., respectively). Accessing the exact energy levels of the triplets in the crystals is beyond the scope of this study, because that would require taking into account the influence of the surrounding molecules. However, it may be assumed that the energies of the ${ }^{3} \mathrm{MLCT}$ and ${ }^{3} \mathrm{IL}$ triplet states are similar in the solids. Emission could thus arise from both triplet states, which is indeed in line with the detection of two distinct lifetimes in both the crystalline and amorphous states. Considering that the small differences may be due to packing effects, comparison between the experimental (Table 1) and calculated (Table S3) values suggests that the ${ }^{3} \mathrm{IL}$ state could be mainly responsible for the PL emission at short wavelengths, which prevails in the crystalline state. In contrast, for the amorphous powder, PL emission in the orange region suggests an increased involvement of the ${ }^{3} \mathrm{MLCT}$ state.

The interplay between the two excited triplet states would therefore be responsible for the bathochromic shift of the PL emission upon grinding the crystalline material, observed for both complexes. However, the question is why the ${ }^{3} \mathrm{IL}$ state would be better stabilised in the crystalline state than in the amorphous state. In fact, due to the bent geometries of $\mathbf{1}$ and 2, the ${ }^{3} \mathrm{IL}$ state has virtually pure IL character, contrary to the complexes studied in the literature, for which the ${ }^{3} \mathrm{IL}$ states have mixed CT and IL character. ${ }^{[17]}$ However, in the present case, the ${ }^{3} \mathrm{IL}$ and ${ }^{3} \mathrm{MLCT}$ states are very different, as shown by their density contour plots (Figure 5). One hypothesis is that some variations in the molecular geometry are necessary to reach the conical intersection that allows transition from one triplet state to the other. In the crystals, such variations are hindered due to intermolecular interactions, and therefore transi-

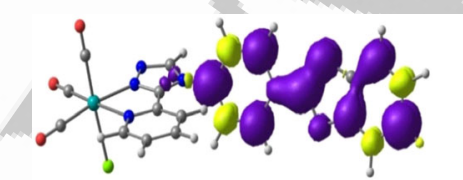

${ }^{3} \mid \mathrm{L}$

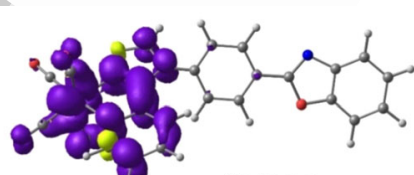

${ }^{3} \mathrm{MLCT}$
Figure 5. Unpaired electron spin density contour plots of the lowest triplet excited states of 2 . tion from ${ }^{3} \mathrm{IL}$ to ${ }^{3} \mathrm{MLCT}$ is prevented to a large extent. By contrast, the release of packing constraints in the amorphous state allows some motion to take place, and the ${ }^{3} \mathrm{MLCT}$ state to be more largely populated. Of course, the most significant molecular motion that is likely to take place is the rotation around the pyta-PBO bond.

The deactivation of these triplet states allows an understanding of the whole phenomenon, which also encompasses the decrease in emission quantum yields and the shortening of phosphorescence lifetimes. These two effects point to a possible mechanism involving competition between radiative and non-radiative relaxation pathways. ${ }^{[18]}$ Evidently, the two triplets have different modes of deactivation. The positions of the orbitals suggest that rotations within the organic ligand are probably the main non-radiative deactivation pathway of ${ }^{3} \mathrm{IL}$. However, the rotational barrier is high (see Table S4 in the Supporting Information) and the crystal packing constraints are strong, so this deactivation pathway would not be efficient, resulting in high PL quantum yields and long lifetimes, as observed in the crystals. The ${ }^{3} \mathrm{MLCT}$ state is not very sensitive to the molecular motions taking place in the organic ligand, and its deactivation should occur through vibrations in the coordination sphere.

The data in Table 1 show that the pristine and ground samples of 1 and $\mathbf{2}$ have the same radiative rate constants, whereas the non-radiative rate constants increase by around fiveand four-fold after the grinding of $\mathbf{1}$ and 2, respectively. Our hypothesis is that the rigid structure of the crystalline state may hinder some vibrational relaxation pathways. Grinding opens this rigid structure to non-radiative relaxation pathways (i.e., vibrational modes). The lower quantum yields and shorter lifetimes may be due to the higher efficiencies of the vibrational relaxation modes compared with radiative ones. The redshifted emission observed for amorphous powders could even be enhanced by the involvement of high-energy vibrational levels of the electronic ground state, resulting in a reduced energy gap.

Emission spectra were also recorded at various temperatures. For pristine powders, only small changes in emission intensity were observed. However, after grinding, the emission intensities of the two complexes decreased markedly at higher temperatures (Figure $6 \mathrm{a}$ and Figure $\mathrm{S} 8$ in the Supporting Information). Heat, which favours vibrational relaxation, has therefore a stronger impact on the ground powders than on the pristine ones. This confirms that grinding opens up vibrational relaxation pathways in the solid complexes, and that the involved triplet excited state is particularly sensitive to this deactivation mode, as expected from the ${ }^{3} \mathrm{MLCT}$ state. Furthermore, FTIR analysis of pristine powders of $\mathbf{2}$ showed well-resolved CO vibrational peaks at 2021, 1934 and $1887 \mathrm{~cm}^{-1} \cdot{ }^{[13 b]}$ Grinding caused a slight broadening of the peak at $2021 \mathrm{~cm}^{-1}$ and a marked broadening of the two other peaks (Figure $6 \mathrm{~b}$ ). These changes in the $\mathrm{CO}$ peaks suggest that the number of vibrations have increased in the amorphous powder, thereby reflecting the existence of new structures in the ground powders, and therefore provide evidence for the opening of the vibrational modes due to the disruption of the rigid crystal struc- 
a

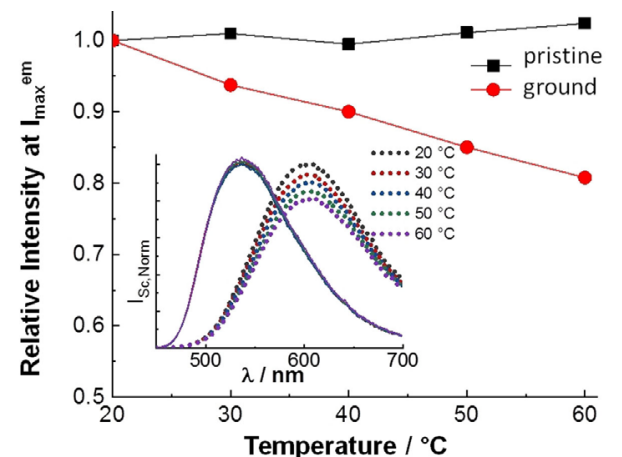

b

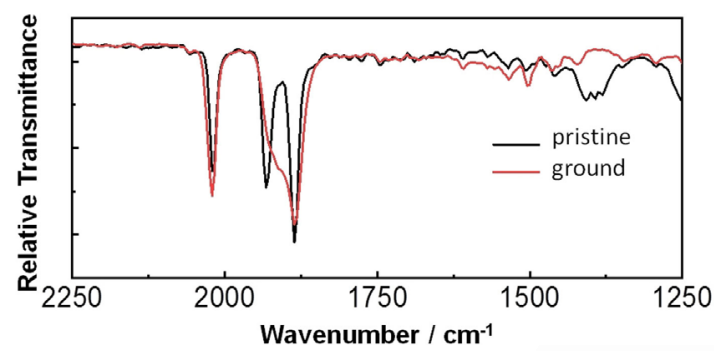

Figure 6. a) Variation of emission intensity at the respective $\lambda_{\max }^{\text {em }}$ of pristine and ground samples of complex $\mathbf{2}$ with increasing temperature. Inset: temperature-dependent emission spectra of the pristine (solid lines) and ground (dotted) samples. b) IR spectra of pristine and ground samples of $\mathbf{2}$.

ture upon grinding. It is well established that the resolution of the bands observed in the IR spectra of rhenium complexes Are the changes for greater clarity ok? $\mathbf{\square}$ is critically dependent on the geometry around the rhenium centre. ${ }^{[19]}$ These changes in the coordination sphere should particularly affect the ${ }^{3}$ MLCT state.

Besides explaining what happens during transitions between crystalline and amorphous states, the same mechanism could account for the spectroscopic differences observed when passing from solution to the solid state. Indeed, free rotation around the pyta-PBO bond would induce total transition from the ${ }^{3} \mathrm{IL}$ state to the lowest-lying ${ }^{3} \mathrm{MLCT}$ state, which is therefore only responsible for phosphorescence emission. Indeed, the phosphorescence emission wavelengths associated with the geometrically optimised ${ }^{3} \mathrm{MLCT}$ triplet states are $608 \mathrm{~nm}$ for 1 and $626 \mathrm{~nm}$ for 2, not far from the experimental values observed in dilute $\mathrm{CH}_{2} \mathrm{Cl}_{2}$ solution, and only one lifetime was detected for each complex. ${ }^{[13 \mathrm{~b}]}$ The $k_{\mathrm{nr}}$ rate constant is almost one order of magnitude higher in solution than in the solid state, which suggests strong vibrational relaxation due to high degrees of freedom in solution and interactions with the solvent. The restriction of molecular vibrations upon transition from solution to the solid state is therefore instrumental for SLE in our $\operatorname{Re}^{\prime}$ complexes, ${ }^{[20]}$ but they must be considered in the context of the interplay between both triplet states, as proposed in Figure 7.

\section{Conclusion}

This work has revealed that SLE and MRL effects are closely linked in our two Rel complexes. The proposed mechanism is original with respect to those commonly reported for organic
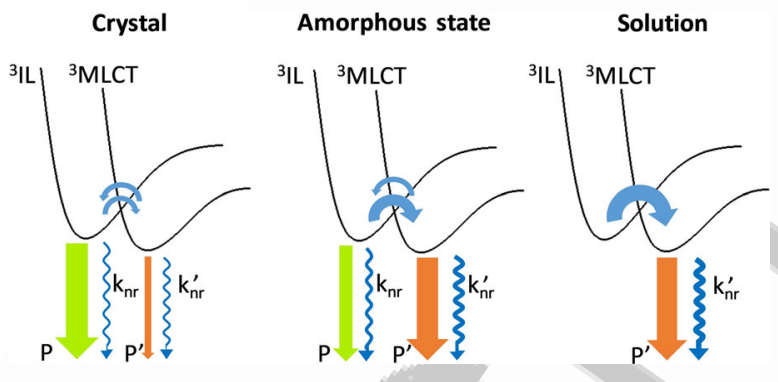

Figure 7. Schematised radiative (straight arrows) and non-radiative (wavy arrows) deactivation pathways of both triplet states in the crystalline and amorphous states, and in solution. The width of the arrows is proportional to the efficiency of the process.

AIE- and MLR-active Change ok? molecules. In the field of transition-metal complexes, the only comparison that seems adequate is with MLR-active Ir"l complexes. ${ }^{[9 c-h]}$ However, these cyclometallated complexes, which are generally cationic, have rather different chemical structures. Their MLR mechanism is based on variations in the intermolecular interactions upon changes in packing ${ }^{[9,, d, e]}$ on the presence of an appended non-planar carbazole unit ${ }^{[9 f, g]}$ and on a trans-to-cis isomerisation of a carbene ligand. ${ }^{[9 h]}$ Interestingly, in the latter case, two types of triplets are involved, but dual emission is far more trivial for Ir ${ }^{\prime \prime \prime}$ complexes than for Re' complexes. It would be instructive to explore whether the original mechanism described here can be applied to the large family of tricarbonyl rhenium(I) complexes, or whether it is related to a particular molecular geometry that generates triplet states with almost pure IL and MLCT character. It must be emphasised that the shift in wavelength (up to $58 \mathrm{~nm}$ ) observed after grinding our complexes compares favourably with that observed for Ir III $^{\prime \prime}$ complexes. The clear colour change from green-yellow to orange, the high PL quantum yields of our compounds and their sensitivity to mechanical sensing make them suitable for practical use. Finally, it is noteworthy that this is the first report that deals with the modulation of SLE emission of $\mathrm{Re}^{\prime}$ complexes through mechanical stimulation, showing that this class of complexes has potential applications in fields requiring MRL-active materials, including security devices and tags.

\section{Acknowledgements}

This work was supported by the European Research Council (ERC StG-715757 MECHANO-FLUO grant to C.A.) and the Chinese Scholarship Council (Ph. D. funding for J.W.). We thank Arnaud Brosseau for his assistance in lifetime measurements and microscopy. The calculations were carried out at the Wroclaw Centre for Networking and Supercomputing, Poland (http://www.wcss.pl).

\section{Conflict of interest}

The authors declare no conflict of interest. 


\section{Keywords: density functional calculations} mechanochemistry $\cdot$ luminescence $\cdot$ rhenium

[1] a) Y. Sagara, S. Yamane, M. Mitani, C. Weder, T. Kato, Adv. Mater. 2016 28, 1073-1095; b) M. Luo, X. Zhou in Mechanochromic Fluorescent Materials: Phenomena, Materials, Applications (Eds.: J. Xu, Z. Chi), Royal Society of Chemistry, Cambridge, 2014, pp. 7-68.

[2] a) Z. Ma, Z. Wang, M. Teng, Z. Xu, X. Jia, ChemPhysChem 2015, 16 $1811-1828$; b) C. Wang, Z. Li, Mater. Chem. Front. 2017, 1, 2174-2194.

[3] a) Z. Chi, J. Xu, in Mechanochromic Fluorescent Materials: Phenomena Materials, Applications (Eds.: J. Xu, Z. Chi), Royal Society of Chemistry, Cambridge, 2014, pp. 163-232; b) C. Wang, Z. Li, in Principles and Applications of Aggregation-Induced Emission (Eds.: Y. Tang, B. Z. Tang), Springer, 口city? $\mathbf{\square}, 2019$, pp. $141-162$.

[4] a) Y. Q. Dong, J. W. Y. Lam, B. Z. Tang, J. Phys. Chem. Lett. 2015, 6, 34293436; b) S. Mukherjee, P. Thilagar, Angew. Chem. 2019, 131, 8004-8014 c) E. Ubba, Y. Tao, Z. Yang, J. Zhao, L. Wang, Z. Chi, Chem. Asian J. 2018 13, 3106-3121; d) J. Zhao, Z. Chi, Z. Yang, Z. Mao, Y. Zhang, F. Ubba, Z. Chi, Mater. Chem. Front. 2018, 2, 1595-1608.

[5] a) Y.-B. Gong, P. Zhang, Y. Gu, J.-Q. Wang, M.-M. Han, C. Chen, W.-J. Zhan, Z.-L. Xie, B. Zhou, Q. Peng, Z.-G. Chi, Z. Li, Adv. Opt. Mater. 2018 6, 1800198; b) L. Zhan, Z. Chen, S. Gong, Y. Xiang, F. Ni, X. Zeng, G. Xie, C. Yang, Angew. Chem. 2019, 131, 17815-17819; c) S. Xu, T. Liu, Y. Mu, Y. F. Wang, Z. Chi, C. C. Lo, S. Liu, Y. Zhang, A. Lien, J. Xu, Angew. Chem. Int. Ed. 2015, 54, 874-878; Angew. Chem. 2015, 127, 888-892.

[6] a) L. Wilbraham, M. Louis, D. Alberga, A. Brosseau, R. Guillot, F. Ito, F. Labat, R. Métivier, C. Allain, I. Ciofini, Adv. Mater. 2018, 30, 1800817 b) Q. Yang, D. Li, W. Chi, R. Guo, B. Yan, J. Lan, X. Liu, J. Yin, J. Mater. Chem. C 2019, 7, 8244-8249; c) J.-A. Li, J. Zhou, Z. Mao, Z. Xie, Z. Yang, B. Xu, C. Liu, X. Chen, D. Ren, H. Pan, G. Shi, Y. Zhang, Z. Chi, Angew. Chem. 2018, 130, 6559-6563.

[7] a) X. Zhang, Z. Chi, Y. Zhang, S. Liu, J. Xu, J. Mater. Chem. C 2013, 1 3376-3390; b) P. Xue, J. Ding, P. Wang, R. Lu, J. Mater. Chem. C 2016, 4, 6688-6706; c) E. Cariati, E. Lucenti, C. Botta, U. Giovanella, D. Marinotto, S. Righetto, Coord. Chem. Rev. 2016, 306, 566-614.

[8] H. Sun, S. Liu, W. Lin, K. Y. Zhang, W. Lv, X. Huang, F. Huo, H. Yang, G. Jenkins, Q. Zhao, W. Huang, Nat. Commun. 2014, 5, 3601.

[9] a) D. Genovese, A. Aliprandi, E. A. Prasetyanto, M. Mauro, M. Hirtz, H. Fuchs, Y. Fujita, H. Uji-I, S. Lebedkin, M. Kappes, L. de Cola, Adv. Funct. Mater. 2016, 26, 5271-5278; b) Y. Dong, J. Zhang, A. Li, J. Gong, B. He S. Xu, J. Yin, S. H. Liu, B. Z. Tang, J. Mater. Chem. C 2020, 8, 894-899; c) Z. Song, R. Liu, Y. Li, H. Shi, J. Hu, X. Cai, H. Zhu, J. Mater. Chem. C 2016, 4, 2553-2559; d) D. Li, G. Li, J. Xie, D. Zhu, Z. M. Su, M. R. Bryce, J. Mater. Chem. C 2019, 7, 10876-10880; e) Y. Wang, T. Yang, X. Liu, G. Li, W. Che, D. Zhu, Z. Su, J. Mater. Chem. C 2018, 6, 12217-12223; f) K.-Y. Zhao, H.-T. Mao, L.-L. Wen, G.-G. Shan, Q. Fu, H.-Z. Sun, Z.-M. Su, J. Mater. Chem. C 2018, 6, 11686-11693; g) G.-G. Shan, H.-B. Li, H.-Z. Sun,
D.-X. Zhu, H.-T. Cao, Z.-M. Su, J. Mater. Chem. C 2013, 1, 1440-1449; h) J. Han, K.-M. Tang, S.-C. Cheng, C.-O. Ng, Y.-K. Chun, Sh.-L. Chan, S.-M. Yiu, M.-K. Tse, V. A. L. Roy, C.-C. Ko, Inorg. Chem. Front. 2020, 7, 786794.

[10] a) C. H. Woodall, C. M. Beavers, J. Christensen, L. E. Hatcher, M. Intissar, A. Parlett, S. J. Teat, C. Reber, P. R. Raithby, Angew. Chem. Int. Ed. 2013, 52, 9691-9694; Angew. Chem. 2013, 125, 9873-9876; b) E. Kwon, J. Kim, K. Y. Lee, T. H. Kim, Inorg. Chem. 2017, 56, 943 -949.

[11] a) B. Hupp, J. Nitsch, T. Schmitt, R. Bertermann, K. Edkins, F. Hirsch, I. Fischer, M. Auth, A. Sperlich, A. Steffen, Angew. Chem. Int. Ed. 2018, 57, 13671-13675; Angew. Chem. 2018, 130, 13860-13864; b) A. Kobayashi, Y. Yoshida, M. Yoshida, M. Kato, Chem. Eur. J. 2018, 24, 14750-14759.

[12] a) H. Ito, M. Marumoto, S. Kurenuma, S. Ishizaka, N. Kitamura, H. Sato, T. Seki, Nat. Commun. 2013, 4, 2009; b) T. Seki, N. Tokodai, S. Omagari, T. Nakanishi, Y. Hasegawa, T. Iwasa, T. Taketsugu, H. Ito, J. Am. Chem. Soc. 2017, 139, 6514-6517.

[13] a) J. Wang, B. Delavaux-Nicot, M. Wolff, S. Mallet-Ladeira, R. Métivier, E. Benoist, S. Fery-Forgues, Dalton Trans. 2018, 47, 8087-8099; b) J. Wang A. Poirot, B. Delavaux-Nicot, M. Wolff, S. Mallet-Ladeira, J. P. Calupitan, C. Allain, E. Benoist, S. Fery-Forgues, Dalton Trans. 2019, 48, $15906-$ 15916.

[14] a) V. Sathish, A. Ramdass, P. Thanasekaran, K.-L. Lu, S. Rajagopal, J. Photochem. Photobiol. C 2015, 23, 25-44; b) L. Ravotto, P. Ceroni, Coord. Chem. Rev. 2017, 346, 62-76; c) J. Shi, L. E. Aguilar Suarez, S.-J. Yoon, S. Varghese, C. Serpa, S. Y. Park, L. Lüer, D Roca-Sanjuán, B. Milián-Medina, J. Gierschner, J. Phys. Chem. C 2017, 121, 23166-23183.

[15] C. Carayon, S. Fery-Forgues, Photochem. Photobiol. Sci. 2017, 16, 10201035.

[16] a) M. Louis, C. P. Garcia, A. Brosseau, C. Allain, R. Métivier, J. Phys. Chem Lett. 2019, 10, 4758-4762; b) L. Polacchi, A. Brosseau, R. Métivier, C. Allain, Chem. Commun. 2019, 55, 14566-14569.

[17] a) A. El Nahhas, C. Consani, A. M. Blanco-Rodríguez, K. M. Lancaster, O. Braem, A. Cannizzo, M. Towrie, I. P. Clark, S. Zàliš, M. Chergui, A. Vlček Inorg. Chem. 2011, 50, 2932-2943; b) R. Baková, M. Chergui, C. Daniel, A. Vlček, S. Zàliš, Coord. Chem. Rev. 2011, 255, 975-989.

[18] B. Valeur, M. N. Berberan-Santos, Molecular Fluorescence. Principles and Applications, 2nd ed., Wiley-VCH, Weinheim, 2013.

[19] L. Suntrup, S. Klenk, J. Klein, S. Sobottka, B. Sarkar, Inorg. Chem. 2017, $56,5771-5783$.

[20] a) Q. Wei, Y. Dai, C. Chen, L. Shi, Z. Si, Y. Wan, Q. Zuo, D. Han, Q. Duan, J. Mol. Struct. 2018, 1171, 786-792; b) M. T. Gabr, F. C. Pigge, Dalton Trans. 2017, 46, 15040-15047; c) T. Tao, H. Fang, Y.-X. Peng, M.-D. Zhang, W. Huang, Inorg. Chem. Commun. 2017, 84, 15-19.

Manuscript received: December 8, 2020

Accepted manuscript online: December 10, 2020

Version of record online: $\mathbf{\square} \mathbf{\square I}, 0000$ 


\section{FULL PAPER}

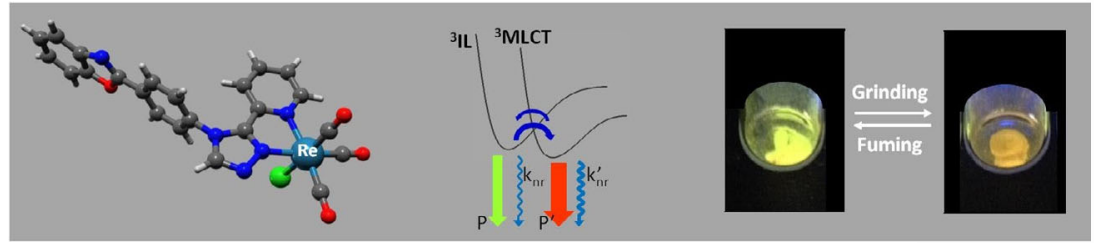

Totally unprecedented! The link between mechanoresponsive luminescence (MRL) and solid-state luminescence enhancement has been explored for two Re' complexes. Remarkably, mechanical stimulation modulates the emission of two low-lying triplet states and opens vibrational modes through the disruption of the rigid crystal structure upon grinding (see figure). This report reveals the unprecedented potential of $\mathrm{Re}^{\prime}$ complexes as MRL-active materials.

\section{Luminescence}

J. P. Calupitan, A. Poirot, J. Wang,

B. Delavaux-Nicot, M. Wolff, M. Jaworska,

R. Métivier, E. Benoist, C. Allain,*

S. Fery-Forgues*

\section{a-}

Mechanical Modulation of the SolidState Luminescence of Tricarbonyl Rhenium(I) Complexes Through the Interplay Between Two Triplet Excited States

Re complexes have unprecedented potential as mechanoresponsive luminescent materials @UnivParisSaclay SPACE RESERVED FOR IMAGE AND LINK

Share your work on social media! Chemistry - A European Journal has added Twitter as a means to promote your article. Twitter is an online microblogging service that enables its users to send and read text-based messages of up to 140 characters, known as "tweets". Please check the pre-written tweet in the galley proofs for accuracy. Should you or your institute have a Twitter account, please let us know the appropriate username (i.e., @accountname), and we will do our best to include this information in the tweet. This tweet will be posted to the journal's Twitter account @ChemEurJ (follow us!) upon online publication of your article, and we recommended you to repost ("retweet") it to alert other researchers about your publication.

Please check that the ORCID identifiers listed below are correct. We encourage all authors to provide an ORCID identifier for each coauthor. ORCID is a registry that provides researchers with a unique digital identifier. Some funding agencies recommend or even require the inclusion of ORCID IDs in all published articles, and authors should consult their funding agency guidelines for details. Registration is easy and free; for further information, see http://orcid.org/.

Dr. Jan Patrick Calupitan

Alexandre Poirot

Dr. Jinhui Wang

Dr. Béatrice Delavaux-Nicot

Dr. Mariusz Wolff

Dr. Maria Jaworska

Dr. Rémi Métivier

Prof. Eric Benoist

Dr. Clémence Allain

Dr. Suzanne Fery-Forgues http://orcid.org/0000-0002-7399-5956

\section{Author Contributions}

J.C. Investigation: Lead; Methodology: Lead; Writing - original draft: Equal; Writing - review \& editing: Supporting

A.P. Investigation: Supporting

J.W. Investigation: Supporting

B.D. Writing - original draft: Supporting; Writing - review \& editing: Supporting

M.W. Investigation: Lead; Writing - original draft: Equal

M.J. Methodology: Equal

R.M. Supervision: Equal; Validation: Equal; Writing - original draft: Supporting; Writing - review \& editing: Supporting

E.B. Supervision: Supporting

C.A. Methodology: Supporting; Resources: Equal; Supervision: Equal; Validation: Equal; Writing - original draft: Equal; Writing - review \& editing: Supporting. 